\title{
Improving the parameterisation of horizontal density gradient in one-dimensional water column models for estuarine circulation
}

\author{
S. Blaise ${ }^{1}$ and E. Deleersnijder ${ }^{2}$ \\ ${ }^{1}$ Université catholique de Louvain, Unité de Génie Civil et Environnemental, Louvain-la-Neuve, Belgium \\ ${ }^{2}$ Université catholique de Louvain, Centre for Systems Engineering and Applied Mechanics, Louvain-la-Neuve, Belgium
}

\begin{abstract}
A new parameterisation of horizontal density gradient for a one-dimensional water column estuarine model, inspired by the first-order finite-difference upwind scheme, is presented. This parameterisation prevents stratification from growing indefinitely, a deficiency usually referred to as "runaway stratification". It is seen that, using this upwind-like parameterisation, the salinity must remain comprised between upper and lower bounds set a priori and that any initial overor under-shooting is progressively eliminated. Simulations of idealised and realistic estuarine regimes indicate that the new parameterisation lead to results that are devoid of the runaway stratification phenomenon, as opposed to previously used models.
\end{abstract}

\section{Introduction}

Estuaries and their regions of freshwater influence (ROFIs) have been studied for a long time. They exhibit strong gradients of several variables: salinity, temperature, plankton and nutrient concentrations can vary over a wide range of values, strongly impacting physical and biological processes. For instance, complex dynamics, influenced by tides and input of freshwater from rivers, have a strong influence on the growth of phytoplankton (Lucas et al., 1998, 1999).

This work focuses on estuarine dynamics, especially on the evolution of stratification. The latter is a key player in vertical mixing, which influences directly the vertical fluxes of heat, salt, momentum and nutrients (Simpson et al., 1990). Many studies were devoted to the evolution of stratification in estuaries. They firstly described in situ observations gathered from field surveys (Sharples and Simpson, 1993; Stacey and Monismith, 1999), showing that the dynamics is mainly

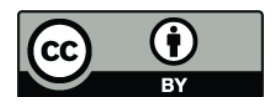

Correspondence to: S. Blaise (sebastien.blaise@uclouvain.be) driven by the tidal flow associated with a density driven circulation generated by an input of freshwater from rivers. This was reproduced in laboratory experiments by Linden and Simpson $(1986,1988)$, who focused on the mechanisms influencing stratification. These mechanisms were described in detail by Simpson et al. (1990). Several models were applied to simulate and understand the evolution of stratification in estuaries. Linear prescriptive models were first used (Simpson et al., 1991; Nunes Vaz and Simpson, 1994; Scott, 2004). Then, several authors turned to one-dimensional water column non linear models (Monismith et al., 1996; Monismith and Fong, 1996; Nunes Vaz and Simpson, 1994; Lucas et al., 1998). Recently, three-dimensional models were used to simulate estuarine flows (Burchard and Baumert, 1998; Hetland and Geyer, 2004; Warner et al., 2005).

One-dimensional non linear models can be very useful to understand and predict the evolution of stratification in an estuary. They are light and simple to build. They require a minimal amount of data and parameters. Furthermore, they generate simple results, which permits to easily understand the key processes and quickly establish diagnoses. However, one common failure of these models is the generation of runaway stratification: when the tidal amplitude is low, stratification tends to grow without bound due to an inadequate parameterisation of horizontal density gradient (Nunes Vaz and Simpson, 1994; Monismith et al., 1996; Warner et al., 2005). This paper shows that simple analytical developments can lead to a new version of the model which keeps stratification under control. It is also seen that, in the long run, the model is insensitive to an unrealistic initial stratification.

Herein we use a one-dimensional finite-element water column model. Such finite-element models and their advantages were described by Hanert et al. (2006, 2007). As mixing is a key player in the evolution of the stratification (Nunes Vaz and Simpson, 1994), we use the Mellor and Yamada level 2.5 turbulence closure (Mellor and Yamada, 1974, 1982; Galperin et al., 1988) which is well suited for the prediction

Published by Copernicus Publications on behalf of the European Geosciences Union. 


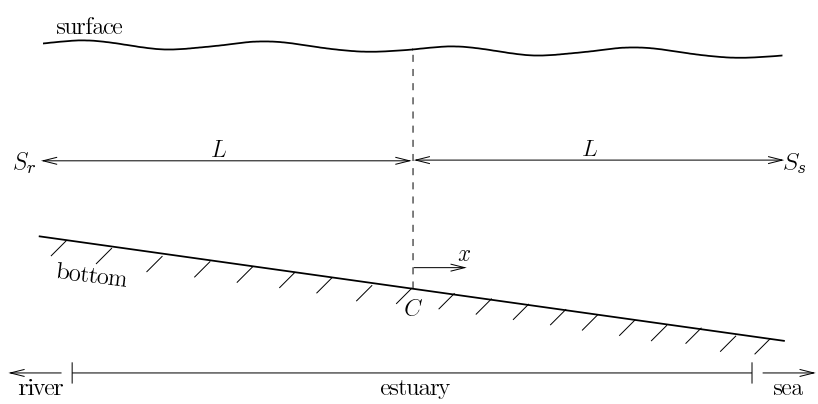

Fig. 1. Physical setting: the stratification is to be simulated in the water column located at point $C$. The latter is located in a region of high salinity gradient. Its order of magnitude is $\left(S_{S}-S_{r}\right) / 2 L$, where $S_{S}$ and $S_{r}$ denote the downstream and the upstream salinity, respectively.

of stratification in estuaries (Nunes Vaz and Simpson, 1994). This turbulence closure was recently implemented using the finite-element method for one-dimensional (Hanert et al., 2006) and three-dimensional (Blaise et al., 2007) models.

The physical setting is described in Sect. 2. Then, in Sect. 3, the model is presented. Two parameterisations of horizontal density gradient, the classical one and a new one, are introduced in Sect. 4 and it is seen rigorously that the new approach prevents stratification from running away. This is illustrated by numerical results in Sect. 5. Section 6 examines the sensitivity of the model to the initial stratification. Finally, conclusions are drawn in Sect. 7.

\section{Physical setting}

We will study the stratification in an estuary, which is generated by the front between freshwater and salty seawater. This front is of a crucial importance for the dynamics of the estuary, notably for the vertical density gradient. Therefore, we will consider a water column located at $C$ in Fig. 1, at a distance $L$ to the sea limit. We assume that the salinity at a distance $L$ upstream of $C$ is of the order of $S_{r}$ and that the salinity at the sea limit is of the order of $S_{s}$. We also assume that $S_{r}$ and $S_{s}$ are constants satisfying the following condition:

$S_{r}<S_{s}$.

In such a configuration, the water velocity is mainly caused by two processes (Simpson et al., 1990):

- The presence of freshwater originating from the river creates a density front with the salty seawater (Fig. 2a). This front induces a circulation, with light freshwater going towards the sea at the surface, and dense water going towards the river near the bottom. Due to the bottom friction, this circulation is reduced near the sea bottom.

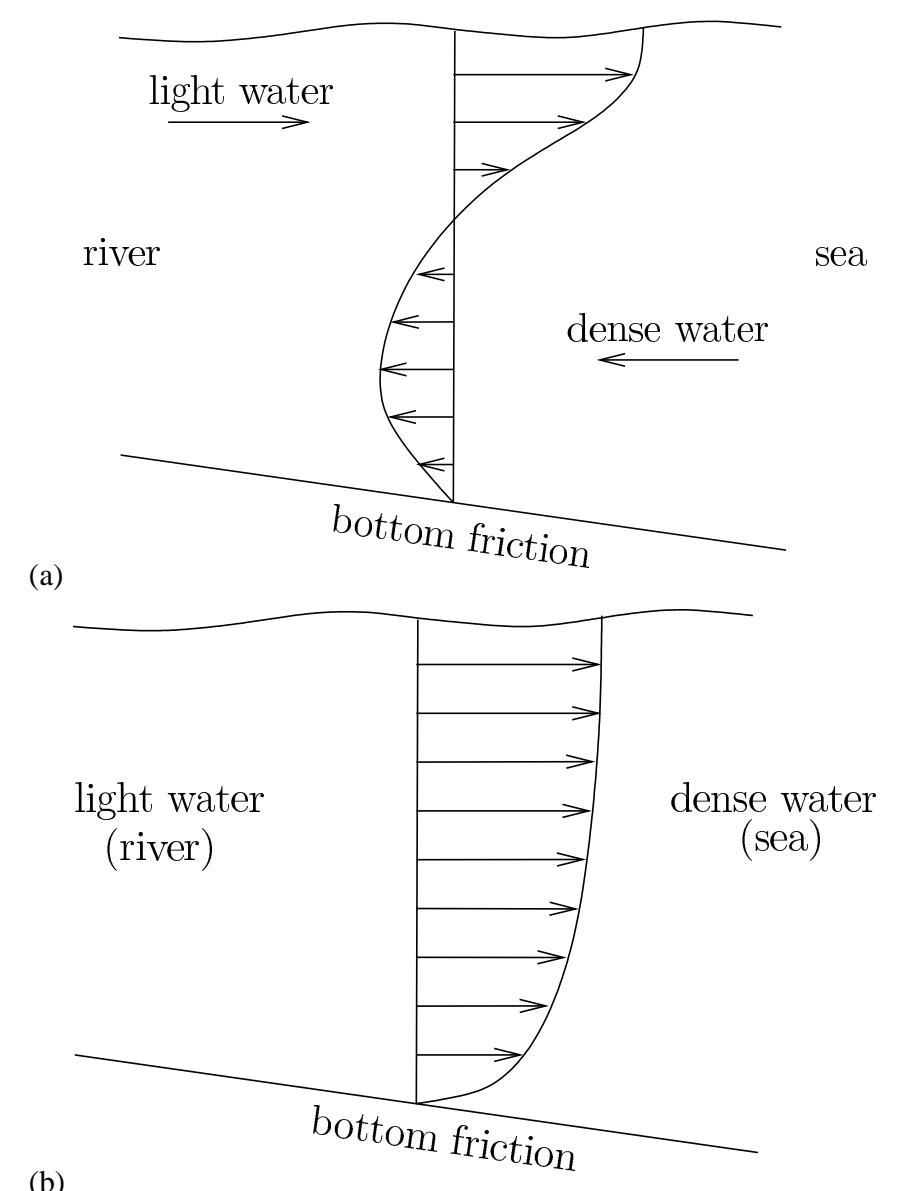

(b)

Fig. 2. Circulation induced (a) by the freshwater input generating a front with the dense seawater and (b) by tides (here at falling tide), as described by Simpson et al. (1990).

- The tidal circulation, influenced by the shear stress due to the bottom friction, generates a logarithmic-like velocity profile (Fig. 2b). This profile induces a transport of freshwater varying over the water column, leading to stratification. The succession of ebbs/floods generates a Strain-Induced Periodic Stratification (SIPS) regime, which can be described as follows: during falling tide, a stable stratification develops, which is reduced by mixing at the end of the falling tide. During rising tide, the salinity profile is unstable and is quickly mixed over the vertical, leading to a non stratified water column. Due to the tidal velocity asymmetry, the velocity profile is different at ebb and flood tides (Jay and Musiak, 1994). The mixing near the bottom is indeed enhanced at flood tides, due to the unstable stratification resulting from the quick displacement of salty water over slow fresh or brackish water (Burchard and Baumert, 1998). This high near-bottom mixing at flood tides leads to higher 
bottom velocities during rising tides than during falling tides, which has the effect of increasing the stratification.

The combination of these processes can generate different flow regimes. If the tides are dominant, the SIPS regime prevails. When the effect of the horizontal density gradient becomes important compared to the tidal effect, the tidal mixing is not sufficient to annihilate the stratification; this stratification strengthens during each tidal cycle, inducing a persistent stratification regime (Lucas et al., 1998). The presence of different non-synchronous tidal components, by generating an alternation of spring/neap tides, can lead to a succession of SIPS and permanent stratification periods (Simpson et al., 1990; Sharples and Simpson, 1993; Nunes Vaz and Simpson, 1994; Monismith et al., 1996).

\section{Model description}

The model used herein is based on that of Lucas et al. (1998) and Monismith et al. (1996). For the flow under study, the impact on density of temperature variations is negligible compared with those of salinity. Therefore, density is assumed to be a function of salinity only and the following equations will be expressed in terms of salinity. As in Lucas et al. (1998) and Monismith et al. (1996), a linear equation of state is adopted:

$\rho=\rho_{0}\left(1+\beta\left(S-S_{0}\right)\right)$,

where $\rho$ and $S$ are the density and the salinity, whose reference values are denoted $\rho_{0}$ and $S_{0}$, respectively; $\beta=7.6 \cdot 10^{-4} \mathrm{psu}^{-1}$ is the salinity expansion coefficient, which is assumed to be constant.

If $x$ is the horizontal coordinate increasing toward the sea, the along-estuary horizontal velocity $u(t, z)$ at location $C$ obeys the following momentum equation:

$$
\frac{\partial u}{\partial t}=-g \frac{\partial \eta}{\partial x}-g \beta \frac{\partial S}{\partial x}\left(-z+\gamma \frac{H}{2}\right)+\frac{\partial}{\partial z}\left(v \frac{\partial u}{\partial z}\right),
$$

where $g, \eta, z$ and $H$ are the gravitational acceleration, the sea surface elevation, the vertical coordinate pointing upwards with its origin at the sea surface and the constant water depth, respectively. The effect of Earth rotation is neglected. The surface stress and bottom velocity are equal to zero. The turbulent viscosity $v$ is calculated by means of the Mellor and Yamada level 2.5 turbulence closure (Mellor and Yamada, $1974,1982)$ implemented in its quasi-equilibrium version (Galperin et al., 1988; Deleersnijder and Luyten, 1994). The surface slope due to the barotropic tides can be represented as

$$
-g \frac{\partial \eta}{\partial x}=\sum_{i} U_{i, \max }\left(\frac{2 \pi}{T_{i}}\right) \cos \left(\frac{2 \pi}{T_{i}} t\right)
$$

in which $T_{i}$ is the tide period and $U_{i, \max }$ the maximum velocity for the $i$-th tidal component. The baroclinic pressure gradient can be divided into two contributions (Lucas et al., 1998; Monismith et al., 1996): a term derived from the horizontal salinity gradient, $g \beta \frac{\partial S}{\partial x} z$, and a term derived from the surface slope generated by the baroclinic flow, $-g \beta \frac{\partial S}{\partial x} \gamma \frac{H}{2}$. The dimensionless coefficient $\gamma$ is to be tuned in such a way that the residual transport is zero, i.e. the average over a tidal cycle of the depth-integrated velocity vanishes. Practically, $\gamma$ is found iteratively to minimize this velocity (Lucas et al., 1998). It is possible to impose a prescribed mean velocity, and in this way take into account the effect of residual runoff from the river (Burchard, 1999), but this was not done in the present paper.

The salinity $S$ obeys the equation

$$
\frac{\partial S}{\partial t}=-u \frac{\partial S}{\partial x}+\frac{\partial}{\partial z}\left(\lambda \frac{\partial S}{\partial z}\right)
$$

where the eddy diffusivity $\lambda$ is obtained from the same turbulence closure model as the eddy viscosity. The surface and bottom salinity fluxes are prescribed to be zero:

$$
\left[\lambda \frac{\partial S}{\partial z}\right]_{z=-H, 0}=0
$$

\section{Parameterisation of the horizontal salinity gradient}

In the previous governing equations, most authors (Nunes Vaz and Simpson, 1994; Lucas et al., 1998; Monismith et al., 1996; Monismith and Fong, 1996) assumed the horizontal salinity gradient to be a constant that was evaluated as follows:

$\frac{\partial S}{\partial x}=\tau$

where $\tau=\frac{S_{s}-S_{r}}{2 L}$. In some situations (e.g. for some idealised studies or when it is in accordance with observations), it is a good choice to prescribe the salinity gradient as a constant. However, this parameterisation has been identified as the cause of the so-called "runaway stratification", a phenomenon in which stratification increases indefinitely (Warner et al., 2005). The salinity reaches values that are no longer comprised in the interval $\left[S_{r}, S_{s}\right]$, which is unacceptable. By annihilating vertical mixing, this overestimated stratification corrupts the computation of the evolution of velocity and water properties.

This complication is related to the variation of the forcing terms over the water column in the momentum Eq. (3). Figure 3 shows that, when averaged over a tidal cycle, the sum of each forcing term present in (3) decreases linearly with depth. This variation over the vertical will lead to a seaward tidally-averaged velocity greater in the upper part of the water column than near the bottom. With such a distribution of the velocity, it is obvious that the use of a constant 


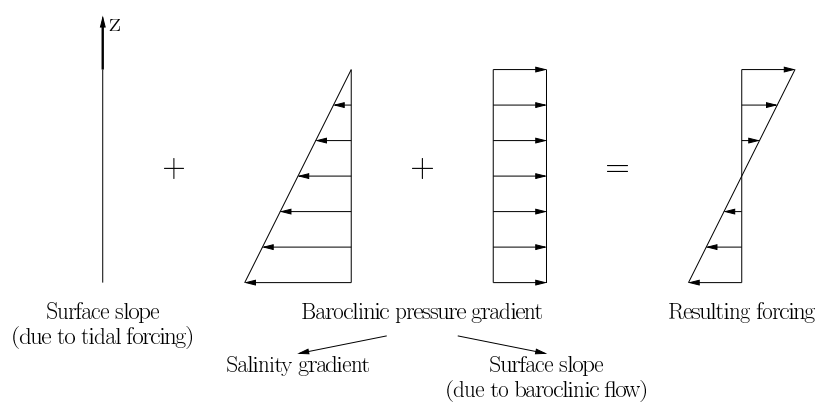

Fig. 3. Vertical profiles of the tidally-averaged forcing terms appearing in the momentum Eq. (3). The resulting forcing varies linearly with depth.

salinity gradient in (5) will inevitably lead to a constantly increasing stratification if mixing is not taken into account. Indeed, at falling tide, the advection of freshwater will decrease with depth whereas, at rising tide, the advection of seawater will increase with depth, causing stratification to grow indefinitely. The turbulent mixing can counterbalance this phenomenon and stabilize the stratification, especially at the end of rising tide when its effect surpasses the effect of advection. At falling tide, the mixing is intense due to the low or unstable stratification, contributing to a non stratified water column. However, if turbulent mixing is not sufficient, the water column will stratify indefinitely.

The apparition of "runaway stratification" can be avoided by using an alternative parameterisation of the horizontal salinity gradient, inspired by the first-order upwind difference scheme:

$\frac{\partial S}{\partial x}= \begin{cases}\frac{S-S_{r}}{L} & \text { if } u \geq 0, \\ \frac{S_{s}-S}{L} & \text { if } u<0 .\end{cases}$

By introducing $u^{+}$and $u^{-}$the positive and negative parts of the longitudinal velocity,

$u^{ \pm}=\frac{u \pm|u|}{2}$,

and by using relation (8), we can rewrite Eq. (5) as

$$
\frac{\partial S}{\partial t}=-u^{+} \frac{S-S_{r}}{L}-\left|u^{-}\right| \frac{S-S_{S}}{L}+\frac{\partial}{\partial z}\left(\lambda \frac{\partial S}{\partial z}\right) .
$$

If the velocity is directed toward the sea $(u>0)$, the first term in the right-hand side of (10) relaxes the salinity to its river value $S_{r}$, the relaxation timescale being $L / u^{+}$. On the other hand, when the velocity is directed toward the river, the salinity is relaxed toward $S_{S}$ with a relaxation timescale equal to $L /\left|u^{-}\right|$.

It is interesting to notice that resorting to this new parameterisation is equivalent to add to the classical formulation (7) a horizontal diffusion term. Indeed, with the parameterisation suggested herein, the horizontal salinity advection may be rewritten as (E. Hanert, personal communication, 2008)

$-u \frac{\partial S}{\partial x}=-u \frac{S_{s}-S_{r}}{2 L}+\frac{|u| L}{2} \frac{S_{s}-2 S+S_{r}}{L^{2}}$.

Clearly, the last term in the equation above may be viewed as the discrete form of the harmonic diffusion operator, the associated diffusivity being $|u| L / 2$.

The interpretation of the role of the first two terms in the right-hand side of salinity Eq. (10) suggests that, whatever the horizontal velocity, the salinity should tend to be comprised in the interval $\left[S_{r}, S_{s}\right]$. In fact, this can be demonstrated rigorously. For an arbitrary large value of $t(t \rightarrow \infty)$, the salinity must obey the following inequalities:

$S_{r} \leq S(t, z) \leq S_{s}$,

implying that stratification cannot grow out of control. We first define the overshooting of the salinity by

$\delta^{+}=\max \left[0, S(t, z)-S_{s}\right]$

So, the overshooting is a positive variable that is equal to $S(t, z)-S_{S}$ if the salinity is greater than its sea value $S_{s}$, and is equal to zero otherwise. Multiplying Eq. (10) by the overshooting and integrating over the height of the water column yields:

$$
\begin{aligned}
& \frac{1}{2} \frac{\mathrm{d}}{\mathrm{d} t} \int_{-H}^{0}\left(\delta^{+}\right)^{2} \mathrm{~d} z= \\
& -\int_{-H}^{0}\left[u^{+} \frac{S-S_{r}}{L}+\left|u^{-}\right| \frac{S-S_{S}}{L}\right] \delta^{+} \mathrm{d} z \\
& \quad-\int_{-H}^{0} \lambda\left(\frac{\partial \delta^{+}}{\partial z}\right)^{2} \mathrm{~d} z .
\end{aligned}
$$

The manipulations leading to this equation are not trivial, but they are of the same type as those of Appendix C of Deleersnijder et al. (2001). All of the terms in the right-hand side of (14) are negative unless the overshooting is zero at every point of the water column. Thus, the quadratic measure of the overshooting tends to zero as time increases, implying that

$\lim _{t \rightarrow \infty} \delta^{+}=0$.

Combining relations (13) and (15) leads to

$S(t, z) \leq S_{S}$ for $t \rightarrow \infty$.

A similar analysis can be performed for the undershooting $\delta^{-}=\max \left[0, S_{r}-S(t, z)\right]$, eventually leading to $S(t, z) \geq S_{r}$ for $t \rightarrow \infty$. Hence, (12) holds valid. QED.

Needless to say, it cannot be seen that, when the classical parameterisation (7) is used, the salinity asymptotically remains within the interval $\left[S_{r}, S_{s}\right]$. 

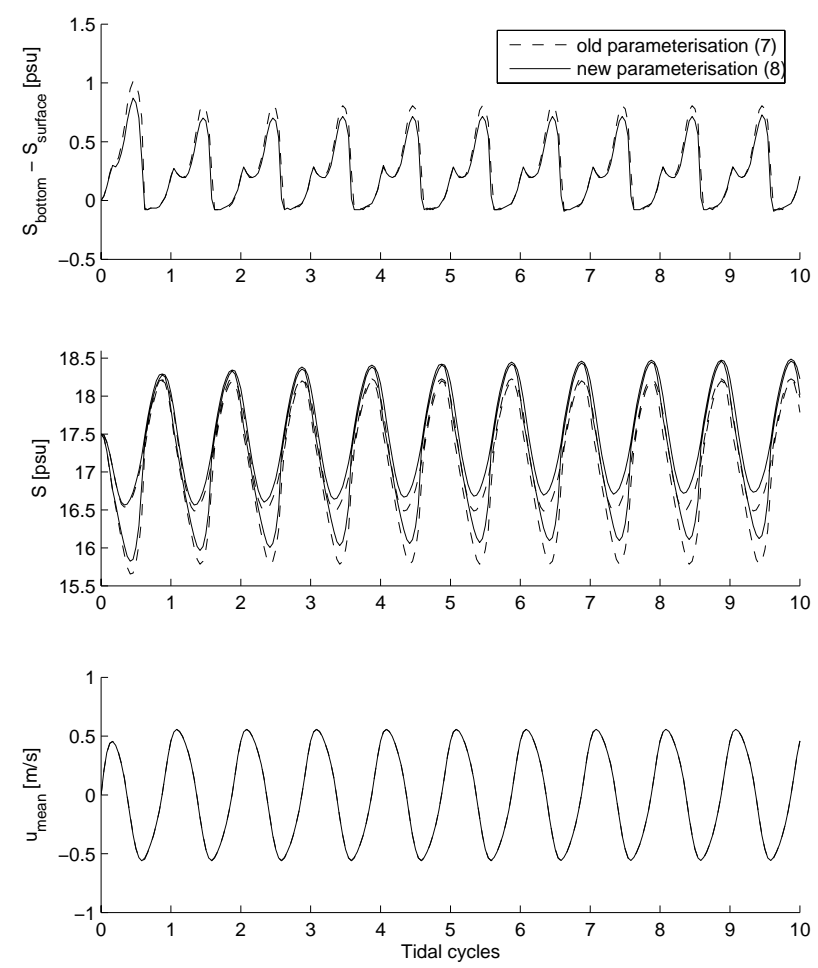

Fig. 4. Simulation of a Strain-Induced Periodic Stratification (SIPS) regime: results obtained using the old (7) (dashed curves) and the new (8) (solid curves) parameterisations of the horizontal salinity gradient. The tidal forcing is characterised by $U_{0, \max }=1 \mathrm{~m} / \mathrm{s}$ and $T_{0}=12 \mathrm{~h}$. The longitudinal salinity gradient is set to $\tau=0.25 \mathrm{psu} / \mathrm{km}$. The bounds of salinity are set to $S_{r}=0 \mathrm{psu}$ and $S_{S}=35$ psu. Upper panel: Evolution of the stratification (difference between bottom salinity and surface salinity). Middle panel: Minimum and maximum values of salinity over the water column. Lower panel: Evolution of the depth-averaged velocity. The latter is similar for both parameterisations.

\section{Model results}

To illustrate the advantages of the parameterisation designed above, we will simulate the situations described in Sect. 2. All of the simulations are achieved using a time-step of 60 seconds. The one-dimensional vertical mesh contains 30 nodes. The main physical parameters are similar to those of Nunes Vaz and Simpson (1994). The water column depth is $15 \mathrm{~m}$, and the values of $S_{r}$ and $S_{s}$ are respectively $0 \mathrm{psu}$ and 35 psu.

We first consider a SIPS regime similar to that of Nunes Vaz and Simpson (1994). There is only one tidal component with a magnitude of $U_{0, \max }=1 \mathrm{~m} / \mathrm{s}$ and a period of $T_{0}=12 \mathrm{~h}$. The longitudinal constant salinity gradient $\tau$ is set to $0.25 \mathrm{psu} / \mathrm{km}$. Figure 4 shows that the SIPS regime is quickly established, with an alternation of stratified/unstratified phases. The tidal mixing at the end of the
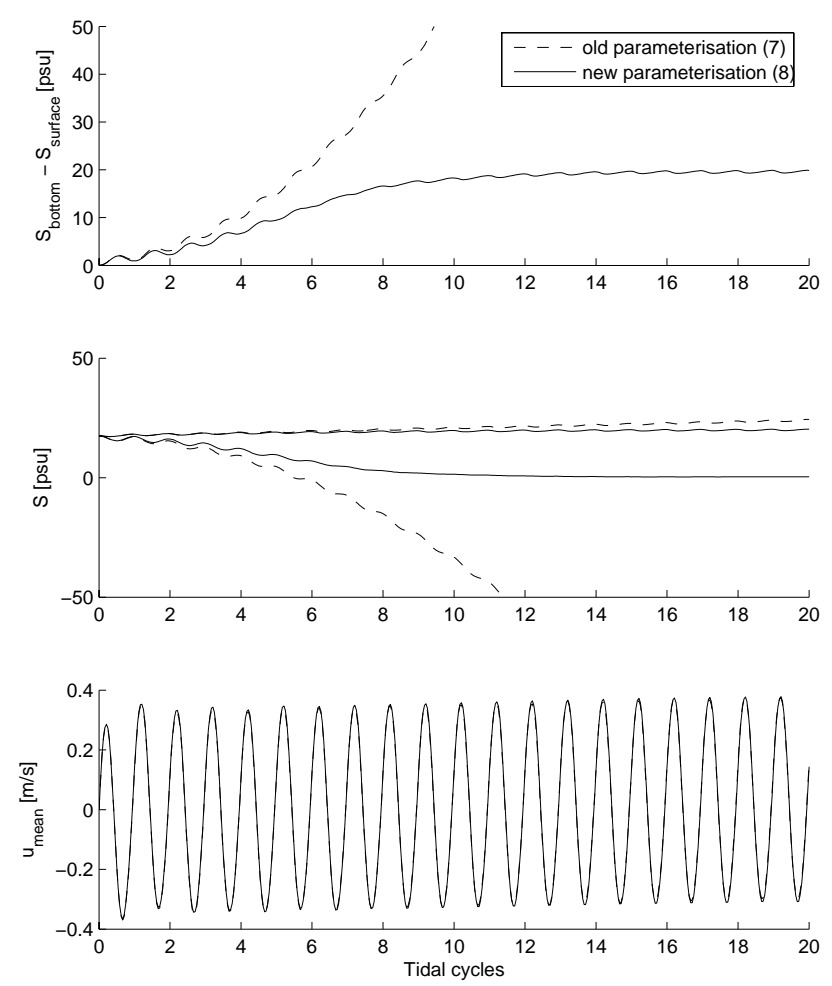

Fig. 5. Simulation of a persistent stratification regime: results obtained using the old (7) (dashed curves) and the new (8) (solid curves) parameterisations of the horizontal salinity gradient. The tidal forcing is characterised by $U_{0, \max }=0.5 \mathrm{~m} / \mathrm{s}$ and $T_{0}=12 \mathrm{~h}$. The longitudinal salinity gradient is set to $\tau=0.3 \mathrm{psu} / \mathrm{km}$. The bounds of salinity are set to $S_{r}=0 \mathrm{psu}$ and $S_{s}=35 \mathrm{psu}$. Upper panel: Evolution of the stratification (difference between bottom salinity and surface salinity). Middle panel: Minimum and maximum values of salinity over the water column. Lower panel: Evolution of the depth-averaged velocity. The latter is similar for both parameterisations.

falling tide is sufficient to annihilate stratification. The latter is very similar using both parameterisations of salinity gradient. However, the constant parameterisation (7) leads to higher peaks of stratification while the latter is limited using the new parameterisation (8). These smaller peaks can be explained by the horizontal diffusion added to the model (11) when we use the new parameterisation of salinity gradient. The mean velocity remains rather insensitive to the used parameterisation.

For the SIPS regime simulated above, the two expressions of the salinity gradient led to rather similar results. This is not always the case, especially if a permanently stratified regime is considered, such as that investigated by Nunes Vaz and Simpson (1994). Accordingly, the tidal amplitude is decreased $\left(U_{0, \max }=0.5 \mathrm{~m} / \mathrm{s}\right)$ to reduce mixing and the longitudinal salinity gradient is increased $(\tau=0.3 \mathrm{psu} / \mathrm{km})$. All the other parameters remain unchanged. Model results are 

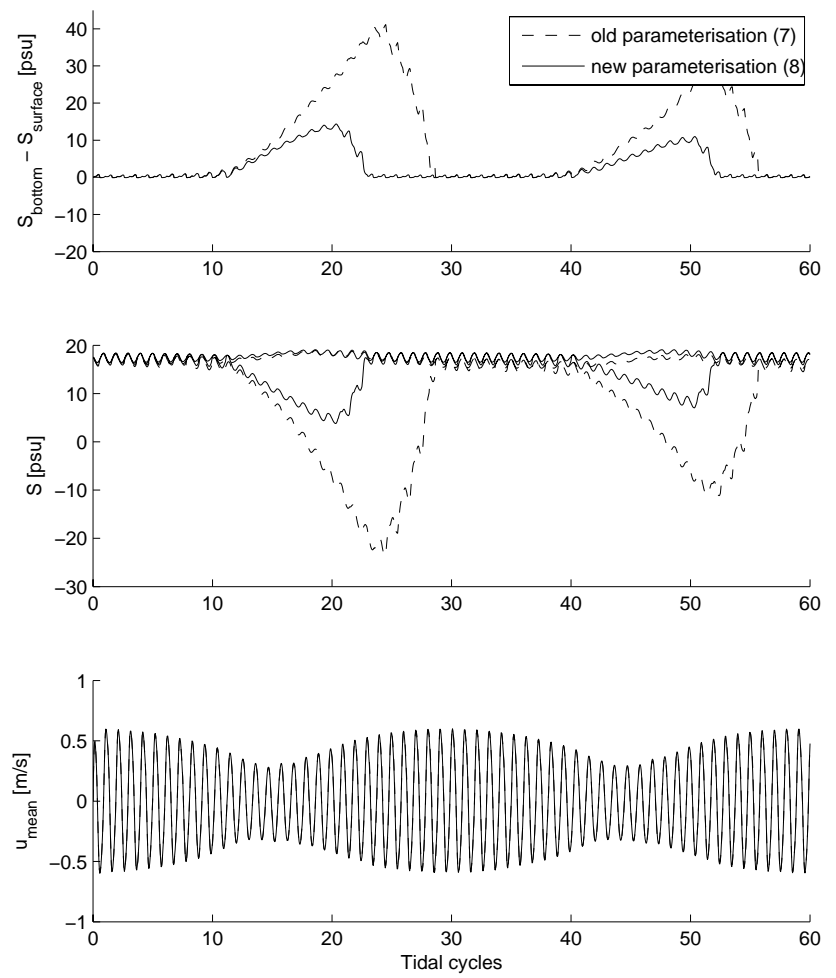

Fig. 6. Simulation of the circulation induced by a succession of spring/neap tides: results obtained using the old (7) (dashed curves) and the new (8) (solid curves) parameterisations of the horizontal salinity gradient. The tidal forcing is characterised by $U_{0, \max }=0.8 \mathrm{~m} / \mathrm{s}, T_{0}=12.42 \mathrm{~h}, U_{1, \max }=0.46 \cdot U_{0, \max }$ and $T_{1}=12 \mathrm{~h}$. The longitudinal salinity gradient is set to $\tau=0.25 \mathrm{psu} / \mathrm{km}$. The bounds of salinity are set to $S_{r}=0$ psu and $S_{S}=35 \mathrm{psu}$. Upper panel: Evolution of the stratification (difference between bottom salinity and surface salinity). Middle panel: Minimum and maximum values of salinity over the water column. Lower panel: Evolution of the depth-averaged velocity. The latter is similar for both parameterisations.

displayed on Fig. 5. Using the classical parameterisation of the horizontal salinity gradient, the stratification grows out of control to unrealistic values exceeding the imposed bounds, which is the deficiency known as "runaway stratification". As demonstrated in Sect. 4, the stratification remains within the imposed limits when we use the new parameterisation. The slight oscillations show that, even when the stratification is high, it is still influenced by tide. While the classical parameterisation (7) gives useless results, the new parameterisation (8) gives qualitatively realistic results for a large number of tidal cycles.

The spring/neap cycles are now simulated by taking into account two tidal components. The first one has an amplitude of $U_{0, \max }=0.8 \mathrm{~m} / \mathrm{s}$ and a period of $T_{0}=12.42 \mathrm{~h}$; while the second component has an amplitude of $U_{1, \max }=0.46 \cdot U_{0, \max }$ and a period of $T_{1}=12 \mathrm{~h}$ (Nunes Vaz and Simpson, 1994).
Using this combination of tidal components, we generate an alternation of spring and neap tides (Fig. 6). We set the longitudinal constant salinity gradient to the value of $\tau=0.25 \mathrm{psu} / \mathrm{km}$. It is shown on Fig. 6 that both parameterisations represent a spring-neap cycle of stratification. During neap tides, the stratification grows until the tidal amplitude increases at spring tides. Then, the stratification weakens and comes back to a SIPS regime. However, the classical parameterisation leads to unrealistic peaks of stratification, with salinity exceeding the limits imposed by the river and sea salinities. This is a common issue when using expression (7) (i.e. Nunes Vaz and Simpson (1994) in which the difference between bottom and surface density grows during neaps as far as $180 \mathrm{~kg} / \mathrm{m}^{3}$ ). This problem does not occur when the new parameterisation is resorted to.

In the last experiment, we simulate a spring-neap cycles regime giving rise to runaway stratification. To this aim, the tidal amplitude is decreased to $U_{0, \max }=0.7 \mathrm{~m} / \mathrm{s}$ and $U_{1, \max }=0.46 \cdot U_{0, \max }$, while the longitudinal constant salinity gradient is increased to $\tau=0.3 \mathrm{psu} / \mathrm{km}$. Figure 7 shows that the classical parameterisation of the horizontal salinity gradient term leads to a stratification which increases unboundedly and then cannot come back to the SIPS regime. During successive tidal cycles, the stratification strengthen to excessively large values. The new parameterisation, by limiting the peak of stratification to acceptable values, permits to come back to the SIPS regime during spring tides which is believed to be consistent with observation (Simpson et al., 1990; Sharples and Simpson, 1993).

\section{Discussion}

We now investigate the impact of the initial conditions, in particular the stratification prescribed at the initial instant. Figure 8a shows the evolution of the stratification using different initial stratifications for the SIPS regime. For each parameterisation, the mixing is able to annihilate the stratification, yielding a SIPS regime. However, the decrease of the stratification is much faster using the new parameterisation. It was demonstrated in Sect. 4 that, even if we have an overshooting or an undershooting in the initial salinity, this excess will be eliminated by the new parameterisation of the horizontal salinity gradient. If the stratification exceeds the upper limit, it cannot strengthen anymore when the new parameterisation is used, whereas the classical parameterisation still generates cycles of increase/decrease of stratification. In a persistent stratification regime (Fig. 8b), using the new parameterisation, the stratification decreases under its upper limit value, and then reaches a regime solution. The solution converges for any initial stratification. This confirms that any overshooting is directly eliminated by that parameterisation. The classical parameterisation, on the other hand, generates a runaway stratification. 

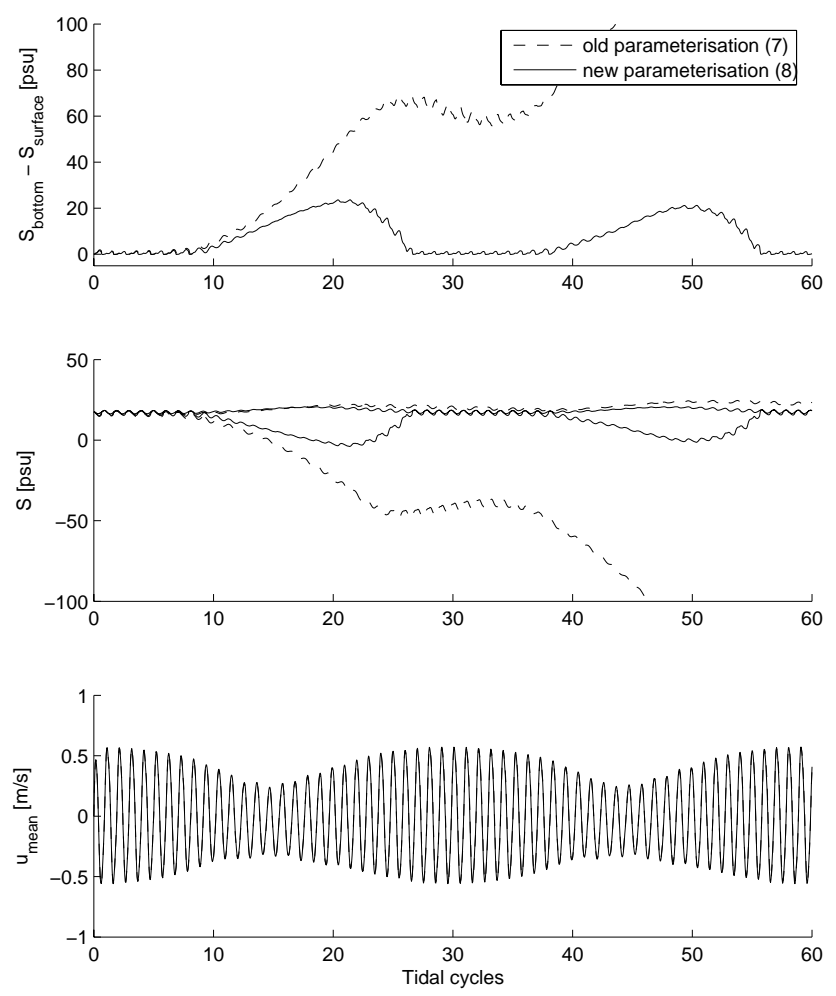

Fig. 7. Simulation of the circulation induced by a of succession spring/neap tides: results obtained using the old (7) (dashed curves) and the new (8) (solid curves) parameterisations of the horizontal salinity gradient. The tidal forcing is characterised by $U_{0, \max }=0.7 \mathrm{~m} / \mathrm{s}, T_{0}=12.42 \mathrm{~h}, U_{1, \max }=0.46 \cdot U_{0, \max }$ and $T_{1}=12 \mathrm{~h}$. The longitudinal salinity gradient is set to $\tau=0.3 \mathrm{psu} / \mathrm{km}$. The bounds of salinity are set to $S_{r}=0$ psu and $S_{S}=35$ psu. Upper panel: Evolution of the stratification (difference between bottom salinity and surface salinity). Middle panel: Minimum and maximum values of salinity over the water column. Lower panel: Evolution of the depth-averaged velocity. The latter is similar for both parameterisations.

By slightly modifying the equations, the present model could also be applied to the simulation of the tidal straining in a Region of Freshwater Influence (ROFI), for which the stratification induced by a gradient of density is also a key process (Visser et al., 1994). The new parameterisation of the salinity gradient should be able to avoid the generation of runaway stratification in a ROFI model, for which this numerical complication can also occur.

\section{Conclusions}

Using simple mathematical developments, a new expression of the horizontal density gradient was developed in order to avoid the phenomenon known as "runaway stratification". This method allows for the simulation of rather realistic flows

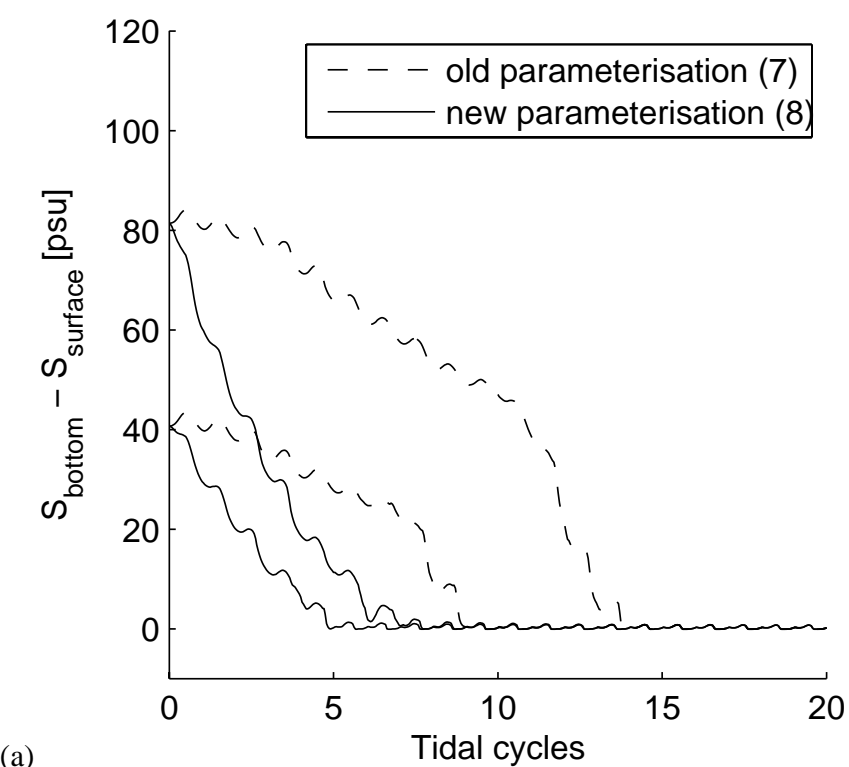

(a)

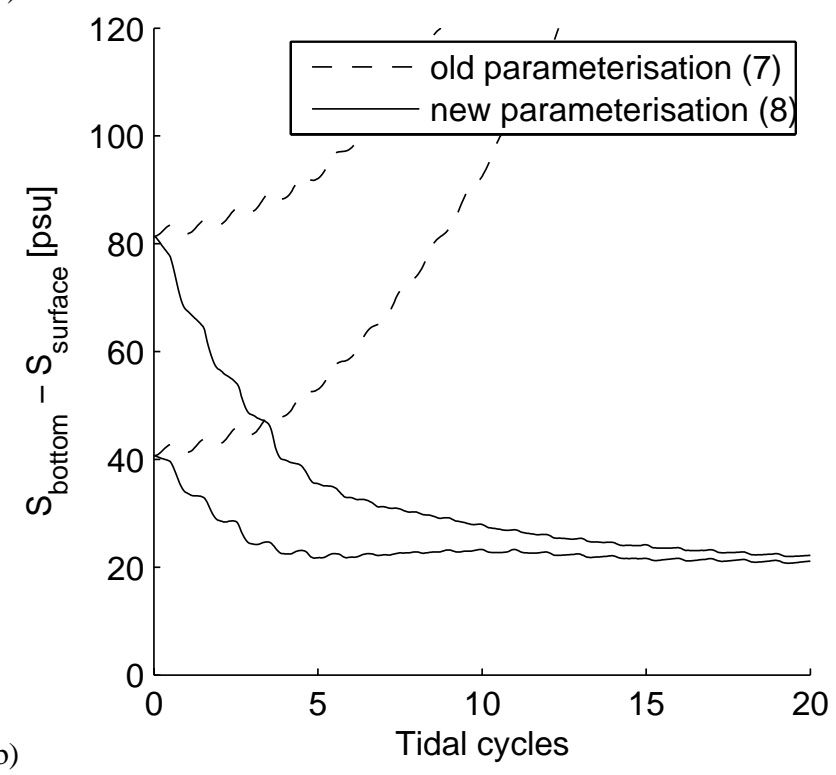

Fig. 8. Sensitivity to the initial stratification: evolution of the stratification (difference between bottom salinity and surface salinity) for the different parameterisations of the horizontal salinity gradient, in the case of a SIPS regime (a) and persistent stratification (b). Two simulation results are showed for both regimes, with initial differences between bottom salinity and surface salinity set to $40 \mathrm{psu}$ and 80 psu.

such as spring/neap cycles without any unrealistic stratification peak. It is guaranteed that no over- or under-shooting will be generated and that any initial over- or under-shooting will progressively disappear. The mathematical method we had recourse to for establishing the properties of the new parameterisation of horizontal salinity gradient may be applied to a wide range of partial differential problems in order 
to derive a priori upper or lower bounds of their solution. This technique is inspired by Lewandowski (1997). To the best of our knowledge, it has been used in a small number of oceanographic studies only (Deleersnijder et al., 2001; Legrand et al., 2006; Gourgue et al., 2007).

Acknowledgements. Sébastien Blaise is a Research fellow with the Belgian Fund for Research in Industry and Agriculture (FRIA). Eric Deleersnijder is a Research associate with the Belgian National Fund for Scientific Research (FNRS). The present study was carried out within the scope of the project "A second-generation model of the ocean system", which is funded by the Communauté Française de Belgique, as Actions de Recherche Concertées, under contract ARC 04/09-316 and the project "Tracing and Integrated Modelling of Natural and Anthropogenic Effects on Hydrosystems" (TIMOTHY), an Interuniversity Attraction Pole (IAP6.13) funded by the Belgian Federal Science Policy Office (BELSPO). This work is a contribution to the development of SLIM, the Second-generation Louvain-la-Neuve Ice-ocean Model (http://www.climate.be/SLIM).

Edited by: E. J. M. Delhez

\section{References}

Blaise, S., Deleersnijder, E., White, L., and Remacle, J.-F.: Influence of the turbulence closure scheme on the finite-element simulation of the upwelling in the wake of a shallow-water island, Cont. Shelf Res., 27, 2329-2345, 2007.

Burchard, H.: Recalculation of surface slopes as forcing for numerical water column models and tidal flow, Appl. Math. Mod., 23, 737-755, 1999.

Burchard, H. and Baumert, H.: The formation of estuarine turbidity maxima due to density effects in the salt wedge. A hydrodynamic process study, J. Phys. Oceanogr., 28, 309-321, 1998.

Deleersnijder, E. and Luyten, P.: On the practical advantages of the quasi-equilibrium version of the Mellor and Yamada level 2.5 turbulence closure applied to marine modelling, App. Math. Mod., 18, 281-287, 1994.

Deleersnijder, E., Campin, J.-M., and Delhez, E. J. M.: The concept of age in marine modelling I. Theory and preliminary model results, J. Mar. Sys., 28, 229-267, 2001.

Galperin, B., Kantha, L., Hassid, S., and Rosati, A.: A quasiequilibrium turbulent energy model for geophysical flows, J. Atmos. Sci., 45, 55-62, 1988.

Gourgue, O., Deleersnijder, E., and White, L.: Toward a generic method for studying water renewal, with application to the epilimnion of Lake Tanganyika, Estuarine, Coastal and Shelf Science, 74, 764-776, 2007.

Hanert, E., Deleersnijder, E., and Legat, V.: An adaptative finite element water column model using the Mellor-Yamada level 2.5 turbulence closure scheme, Ocean Model., 12, 205-223, 2006.

Hanert, E., Deleersnijder, E., Blaise, S., and Remacle, J.-F.: Capturing the bottom boundary layer in finite element ocean models, Ocean Model., 17, 153-162, 2007.

Hetland, R. D. and Geyer, W. R.: An idealized study of the structure of long, partially mixed estuaries, J. Phys. Oceanogr., 34, $2677-$ 2691, 2004.
Jay, D. A. and Musiak, J. D.: Particle trapping in estuarine tidal flows, J. Geophys. Res., 99, 445-461, 1994.

Legrand, S., Deleersnijder, E., Hanert, E., Legat, V., and Wolanski, E.: High-resolution, unstructured meshes for hydrodynamic models of the Great Barrier Reef, Australia, Estuarine, Coastal and Shelf Science, 68, 36-46, 2006.

Lewandowski, R.: Analyse Mathématique et Océanographie, Masson, Paris, 281 p., 1997.

Linden, P. F. and Simpson, J. E.: Gravity-driven flows in a turbulent fluid, J. Fluid Mech., 172, 481-497, 1986.

Linden, P. F. and Simpson, J. E.: Modulated mixing and frontogenesis in shallow seas and estuaries, Cont. Shelf Res., 8, 1107-1127, 1988.

Lucas, L. V., Cloern, J. E., Koseff, J. R., Monismith, S. G., and Thompson, J. K.: Does the Sverdrup critical depth model explain bloom dynamics in estuaries?, J. Mar. Res., 56, 375-415, 1998.

Lucas, L. V., Koseff, J. R., Cloern, J. E., Monismith, S. G., and Thompson, J. K.: Processes governing phytoplankton blooms in estuaries. I: The local production-loss balance, Marine Ecology Progress Series, 187, 1-15, 1999.

Mellor, G. L. and Yamada, T.: A hierarchy of turbulence closure models for planetary boundary layers, J. Atmos. Sci., 31, 17911806, 1974.

Mellor, G. L. and Yamada, T.: Development of a turbulence closure model for geophysical fluid problems, Review of Geophysics and Space Physics, 20, 851-875, 1982.

Monismith, S. G. and Fong, D. A.: A simple model of mixing in stratified tidal flows, J. Geophys. Res., 101, 28 583-29 595, 1996.

Monismith, S. G., Burau, J. R., and Stacey, M. T.: San Francisco Bay: The Ecosystem, chap. Stratification dynamics and gravitational circulation in Northern San Francisco Bay, pp. 123153, American Association for the Advancement of Science, San Francisco, 1996.

Nunes Vaz, R. A. and Simpson, J. H.: Turbulence closure modeling of estuarine stratification, J. Geophys. Res., 99, 16143-16160, 1994.

Scott, C. F.: A prescriptive bulk model of periodic estuarine stratification driven by density currents and tidal straining, Environmental Modeling and Assessment, 9, 13-22, 2004.

Sharples, J. and Simpson, J. H.: Periodic frontogenesis in a region of freshwater influence, Estuaries, 16, 74-82, 1993.

Simpson, J. H., Brown, J., Matthews, J., and Allen, G.: Tidal Straining, Density Currents, and Stirring in the Control of Estuarine Stratification, Estuaries, 13, 125-132, 1990.

Simpson, J. H., Sharples, J., and Rippeth, T. P.: A prescriptive Model of Stratification Induced by Freshwater Runoff, Estuarine, Coastal and Shelf Science, 33, 23-35, 1991.

Stacey, M. T. and Monismith, S. G.: Observations of turbulence in a partially stratified estuary, J. Phys. Oceanogr., 29, 1950-1970, 1999.

Visser, A. W., Souza, A. J., Hessner, K., and Simpson, J. H.: The effect of stratification on tidal current profiles in a region of freshwater influence, Oceanol. Acta, 17, 369-381, 1994.

Warner, J. C., Geyer, W. R., and Lerczak, J. A.: Numerical modeling of an estuary: a comprehensive skill assessment, J. Geophys. Res., 110, C05001, doi:10.1029/2004JC002691, 2005. 